\title{
DIAGNÓSTICO DA UTILIZAÇÃO DA REALIDADE VIRTUAL E REALIDADE AUMENTADA POR ALUNOS DO CURSO DE MEDICINA EM PRESIDENTE PRUDENTE
}

\author{
Adriane Cavichiolli ${ }^{1}$, Elaine Parra Affonso ${ }^{1}$, Eliane Vendramini de Oliveira ${ }^{1,2}$ \\ ${ }^{1}$ Análise e Desenvolvimento de Sistemas - Faculdade de Tecnologia de Presidente Prudente - Presidente Prudente-SP. \\ ${ }^{2}$ Ciência da Computação e Análise e Desenvolvimento de Sistemas - Faculdades Adamantinenses Integradas - \\ Adamantina-SP. E-mail: adriane.cavichiolli@fatec.sp.gov.br
}

\section{RESUMO}

Este trabalho aborda o uso de Realidade Virtual e Realidade Aumentada na medicina para estudo e treinamento de profissionais que são enriquecidos por essa tecnologia, a qual oferece um nível de abstração maior que explicações em salas de aula onde, geralmente, os alunos são submetidos a aulas teóricas e cansativas. A cada dia aparecem novas descobertas na área de Realidade Virtual possibilitando novas aplicações. Neste trabalho será apresentado um levantamento sobre o conhecimento e uso da Realidade Virtual e Realidade Aumentada através de questionário distribuído aos estudantes do curso de medicina da cidade de Presidente Prudente, onde serão apresentados os resultados obtidos.

Palavras-chave: realidade virtual, realidade aumentada, treinamento, simulação, medicina.

\section{INTRODUÇÃO E OBJETIVO}

A Realidade Virtual, ao longo de sua evolução vêm contribuindo para o surgimento de inovações tecnológicas importantes para o ambiente empresarial e de inovações sociais capazes de gerar benefícios efetivos para a população. A variação de Realidade Virtual (RV) é chamada de Realidade Aumentada (RA) que faz uso da mesma tecnologia. Na RV o ambiente virtual se sobressai ao ambiente real, enquanto que na RA o senso de presença do usuário é mantido no mundo real, que em nenhum momento deixa de sentir o ambiente real que se sobressai ao virtual (KIRNER; SISCOUTO, online, 2007).

A RV é uma tecnologia que tem sido efetivamente aplicada em muitas áreas incluindo protótipos, visualização de dados, simulações, treinamento em tarefas de risco, comunicação, entretenimento e muito usada na área médica para treinamento de novos profissionais e de cirurgiões para adquirir novas experiências (CARDOSO; LAMOUNIER Jr., online, 2009).

A Realidade Virtual e Realidade Aumentada conseguem proporcionar inúmeros benefícios na medicina, onde é muito utilizada, reproduzindo até seres humanos de forma virtual para que se possam estudar partes dos seus corpos que só poderiam ser estudadas em corpos sem vida. A extensão de aplicações em que abrange essa área possibilita que estudiosos e pesquisadores descubram novos equipamentos que auxiliem o uso dessa tecnologia nos treinamentos que 
certamente constituem a categoria mais explorada quando se trata de aplicações de RV e RA na área da saúde.

Este trabalho tem como objetivo apresentar uma explanação geral sobre Realidade Virtual e Realidade Aumentada, algumas aplicações como exemplo para melhor entendimento, tendo como foco principal as aplicações de ambientes virtuais na área médica e apresentar os resultados de um levantamento realizado sobre o conhecimento e uso da Realidade Virtual e Realidade Aumentada, por estudantes de medicina da cidade de Presidente Prudente.

\section{Realidade Virtual na Medicina}

O tratamento de fobia também foi beneficiado usando técnicas de RV que proporcionam aos pacientes, maior segurança, menos perigo, pois são aplicadas dentro do consultório com uma exposição gradativa de acordo com o desenvolvimento do tratamento (CARDOSO; LAMOUNIER Jr., online, 2009). As pessoas que tem algum tipo de trauma ou medo por altura, profundidade, animais, insetos e outras fobias, são expostas gradativamente a cada sessão a essas situações dentro de um ambiente virtual e monitoradas a pressão arterial e os batimentos cardíacos dos pacientes (SILVANO et al., online, 2000).

Com o avanço da tecnologia de RA, médicos descobriram por acaso que quando os raios infravermelhos incidem sob a pele, as veias aparecem como se a pele ficasse transparente. Após estudos, descobriu-se que adaptando os equipamentos seria possível utilizar a descoberta para o tratamento de varizes e que não deixam cicatrizes, pois não é feito nenhum corte, apenas aplicam o laser. Muitos pacientes idosos, crianças e pacientes obesos também poderiam ser beneficiados por essa tecnologia facilitando na hora de achar uma veia (PONTUAL; MENEZES, online, 2009).

A educação médica encara diversos desafios atualmente que estão relacionados à formação de futuros médicos e atualização de profissionais por causa da dificuldade de contato direto com pacientes que não pode ocorrer sem a supervisão de médicos e professores. No caso de acontecer erro médico podem ocasionar problemas de saúde para os pacientes e aos profissionais médicos, problemas éticos e judiciais. Para isso, estudos de ambientes virtuais estão sendo desenvolvidos, com o propósito de impulsionar o crescimento do número e da qualidade dos procedimentos médicos e diminuir a necessidade de contatos reais entre futuros médicos e pacientes nas fases iniciais do aprendizado. Módulos virtuais para o estudo de anatomia diminuem a necessidade do uso de cadáveres e possibilita a observação dos órgãos em 
funcionamento diminuindo os efeitos psicológicos gerados nos estudantes pelo contato com cadáveres reais (NUNES et al., online, 2007).

A utilização de computadores na medicina, estudos e o desenvolvimento de aplicações utilizando Realidade Virtual e Realidade Aumentada é uma tendência para essa área. A necessidade de gerar sensações próximas às reais exige ambientes virtuais realísticos para obter respostas às ações que permitam ao usuário ter a sensação de um fato real, e, para isso existem alguns equipamentos utilizados em aplicações de RV na área médica, tais como dispositivos hápticos, luvas e capacetes de visualização (NUNES et al., online, 2007).

A grande preocupação da comunidade médica é com a recuperação dos pacientes após procedimentos médicos e por isso a classe médica apresenta grande interesse nas cirurgias minimamente invasivas onde a incisão é feita o menor possível e a recuperação do paciente é mais rápida proporcionando menos gastos, liberando leitos mais rapidamente com serviços e resultados mais eficientes.

Segundo Greenleaf (2005 apud SOUZA, online, 2007) a Realidade Virtual é aplicada em sete áreas na medicina: treinamento e planejamento cirúrgicos; educação e treinamento não cirúrgicos; fusão de objetos virtuais com imagens reais; reabilitação facilitada por realidade aumentada; soluções para diminuições físicas; telecirurgia e telemedicina; testes de pacientes e intervenção comportamental.

Já foram desenvolvidos diversos simuladores nessa área que auxiliam em procedimentos que só poderiam ser feitos em cadáveres reais com um número de vezes limitados para o procedimento, mas com os simuladores o procedimento pode ser repetido por centenas de vezes e com a vantagem de ser gravado para posterior estudo e reavaliação do profissional (NUNES; MACHADO; COSTA, online, 2009).

$\mathrm{O}$ ensino e treinamento médico através da RV possibilitam o estudo de anatomia e simulação de operações principalmente para o treinamento de cirurgia vídeo-laparoscópica, planejamento pré-operatório e no suporte intra-operatório. São utilizados modelos virtuais com imagens tridimensionais que possibilitam uma avaliação dos órgãos internos com a vantagem da não limitação do tempo e do recurso da semitransparência que possibilita a avaliação da relação entre os órgãos e sua descrição minuciosa. O uso da RV para ensino pode atingir áreas em que os profissionais atualmente não conseguem atingir, pois algumas pessoas têm maior facilidade de aprendizado apenas pela observação visual o que muitas vezes uma explicação verbal não consegue atingir os objetivos para o real entendimento (MONTERO, ZANCHET, online, 2003). 
Segundo Cardoso e Lamounier Jr. (2009), a cada ano morrem mais pessoas de erros médicos do que de acidentes nas estradas, de câncer de mama ou AIDS. A principal razão é a inexperiência de médicos iniciantes, inexperiência sobre novas técnicas cirúrgicas e situações raras presenciadas pelos médicos. Uma das razões pela qual isso ocorre é a falta de investimento em educação e treinamento direcionados a esses profissionais. Com grande avanço a medicina tem sido auxiliada com recursos de Realidade Virtual e Aumentada para o treinamento em modelos anatômicos que através de micro câmeras direcionam os equipamentos e os utensílios usados pelos médicos durante cirurgias, terapias diversas e reabilitação (CARDOSO; LAMOUNIER Jr, online, 2009).

A medicina tem por objetivos principais salvar ou melhorar a qualidade de vida dos seres humanos e para isso procura sempre atualizações na área médica e também tecnologias novas com o intuito de melhorar e aprimorar o aprendizado e treinamento de profissionais novos e também os que estão na área há algum tempo. Alguns treinamentos podem influenciar nas decisões médicas, causar menos desconforto e dor aos pacientes, facilitar diagnósticos e também melhorar a destreza e manuseio de utensílios médicos. A Realidade Virtual possui uma vasta aplicabilidade na área médica e por isso pesquisadores têm se interessado e desenvolveram alguns simuladores para auxiliar e facilitar o profissional da medicina.

De acordo com Skinovsky, Chibata e Siqueira (online, 2008), já existe no mercado simuladores para: cirurgias ortopédicas; procedimentos endoscópicos variados envolvendo cirurgia vascular, urologia, aparelho digestório; cirurgias videolaparoscópicas que inclui sutura, hemostasia, dissecação; colecistectomia; gastrofundoplicatura; hernioplastia (inguinal e incisional); gastroplastia, entre outras.

\section{METODOLOGIA}

Em relação à metodologia adotada para a execução deste trabalho, foi utilizada como procedimento técnico a pesquisa aplicada, pois se baseia em dados de alunos do curso de Medicina de Presidente Prudente, suficientemente constatados, inferindo uma verdade sobre a utilização de ferramentas de realidade virtual nas aulas, entre eles os simuladores. Para alcançar e expor os resultados deste trabalho foi aplicado um questionário, para que os alunos relatassem sua opinião sobre seu conhecimento e sobre o uso durante as aulas de tecnologias como RV, RA e simuladores médicos usados para ensino de anatomia, simulação de operações, planejamento pré-operatório e no suporte intra-operatório. O questionário foi respondido por oitenta alunos do 
curso de medicina, do primeiro ao décimo primeiro termo, selecionados pelos professores da Faculdade de Medicina de Presidente Prudente. A forma de abordagem do tema em questão foi quantitativa, pois o resultado do questionário traduz em números as opiniões, informações, experiências e expectativa dos alunos em relação ao conhecimento e uso de técnicas de realidade virtual na medicina.

\section{RESULTADOS}

A seguir são apresentados os dados sobre o conhecimento dos estudantes com relação às tecnologias Realidade Virtual e Realidade Aumentada que tiveram acesso ao questionário que foi distribuído aos alunos do curso de medicina da cidade de Presidente Prudente e os resultados obtidos.

O questionário foi aplicado para alunos do curso de medicina da Unoeste de Presidente Prudente. A média de idade entre eles é de 22 anos sendo que o aluno mais novo tem 18 anos e o mais velho tem 45 anos.

Em relação ao conhecimento da tecnologia Realidade Virtual $42 \%$ dos alunos responderam que já ouviram falar sobre Realidade Virtual e 58\% responderam que não.

A pesquisa realizada com os estudantes indica que $81 \%$ nunca ouviram falar sobre Realidade Aumentada e apenas 19\% dos alunos conhecem a tecnologia.

Em relação ao conhecimento dos alunos quanto ao uso de RV e RA em simuladores na medicina para treinamento médico, a maioria (78\%) respondeu que não tem conhecimento do uso de RA e RV em simulações médicas, e a minoria (22\%) responderam que tem conhecimento.

$\mathrm{Na}$ questão em que perguntava se algum professor já utilizou ou apresentou softwares de Realidade Virtual ou Realidade Aumentada nas aulas, 91\% dos alunos responderam que não e apenas $9 \%$ dos alunos responderam que sim, que já teve contato com a tecnologia durante as aulas.

Na questão que perguntava se algum professor já utilizou ou apresentou softwares de Realidade Virtual ou Realidade Aumentada nas aulas, os alunos que responderam sim, também puderam responder em quais disciplinas foram usados estes softwares e $33 \%$ responderam Gastrologia, 16\% responderam Biologia Molecular, 17\% responderam Medicina Legal, 17\% responderam Congresso de Cardiologia e 17\% responderam Ginecologia e Obstetrícia.

Sobre o conhecimento de algum software de Realidade Virtual ou Realidade Aumentada usado para simulações em treinamento médico, 3\% dos alunos responderam que sim, tem 
conhecimento de softwares usados para simulações, 1\% não responderam a essa questão e $96 \%$ responderam que não conhecem nenhum software usado em simuladores como mostra a Figura 6. Dos alunos que responderam sim, a maioria não sabia o nome do software e citaram apenas as Simulações de casos clínicos na internet.

Quanto ao conhecimento dos alunos de outras áreas que utilizam software de RV e RA além da medicina, $85 \%$ responderam que não conheciam nenhuma outra área que faz uso desses softwares, $15 \%$ responderam que tinham conhecimento sim de outras áreas que utilizam a RV e RA além da medicina.

Com relação ao conhecimento por parte dos alunos de outras áreas além da medicina que utilizam software de RV e RA mostrou que a maioria das áreas citadas tem 6\%, áreas como a Fisioterapia, Física Nuclear, Engenharia Mecânica, Direito, Microbiologia, Odontologia, Automóveis, Embarcações marítimas, Treinamento de piloto de aeronave, Exército americano e Treinamento militar. A área de Engenharia civil tem uma fatia de $12 \%$ e a Biomedicina e Medicina veterinária com $11 \%$ e foram as mais indicadas pela maioria dos alunos.

Com relação a quais disciplinas os alunos do curso de medicina gostariam de trabalhar com software de RV e RA foram citadas várias, tais como: Anatomia Topográfica, Biofísica, Clínica médica, Disciplinas que evoluem na prática médica, Escopias, Farmacologia, Fisiopatologia, Medicina Legal, Pediatria, Procedimentos endoscópicos, Psicologia, Psiquiatria, Urologia e nenhuma ( $1 \%$ dos alunos citaram essas disciplinas). Com $2 \%$ das indicações ficaram as disciplinas: Cardiologia, Emergência médica, Ética médica, Fundamentos do diagnóstico médico, Gastrologia, Genética, Histologia, Otorrinolaringologia, Ortopedia, Patologia, Programa de Aproximação Progressiva à Prática, Pneumologia. Com 3\% das indicações ficaram as disciplinas: Hematologia, Radiologia e Todas. Com $4 \%$ de indicações ficaram as disciplinas: Fisiologia, Neuroanatomia, Neurologia e Oncologia. Com $8 \%$ das indicações ficaram as disciplinas de Anatomia e Cirurgias. Uma porcentagem maior com $11 \%$ disseram que não sabiam sobre o assunto e $16 \%$ não respondeu essa questão.

\section{DISCUSSÃO}

De acordo com as respostas obtidas com o questionário distribuído entre os alunos do curso de medicina de Presidente Prudente, ficou claro que os alunos dos primeiros termos não tem conhecimento sobre os simuladores e os benefícios que a Realidade Virtual e a Realidade Aumentada podem oferecer na área médica, mas os alunos dos termos mais avançados começam 
a ter algum contato com a tecnologia apesar de ser bem superficial, tanto que alguns alunos tiveram contato mas não sabem que tipo de tecnologia estavam usando. O conhecimento de alguns alunos de uma mesma turma e a total falta de conhecimento de outros alunos que motivou essa conclusão.

A RV e RA precisam ser mais divulgadas para que possa haver maior interesse em pesquisas na área com o intuito de desenvolvimento de dispositivos mais acessíveis para o usuário e promover o barateamento da tecnologia.

\section{CONCLUSÃO}

A Realidade Virtual é uma tecnologia tridimensional desenvolvida para ambientes gráficos, muito promissora nos dias de hoje. Ela pode promover inúmeros benefícios aos profissionais para estudo e treinamento de disciplinas que necessitam muito mais do que livros e professores porque com o uso de aplicações de Realidade Virtual e Realidade Aumentada é possível simular ambientes específicos da área de medicina. Além disso, é possível a manipulação do ambiente virtual através de dispositivos da área que proporcionam ao usuário interação em tempo real.

A utilização dessa tecnologia para fins educacionais e principalmente treinamento médico pode não só auxiliar o profissional, mas promover um conhecimento muito maior devido ao contato com as imagens que segundo essa pesquisa e alguns autores, o ser humano tem muito mais facilidade em guardar informações quando tem contato direto com o objeto em estudo.

Foi um grande desafio conseguir que os alunos de Medicina encontrassem disponibilidade de tempo para responder ao questionário proposto apesar de ser uma turma bem grande de jovens que fazem o curso de medicina em Presidente Prudente.

$\mathrm{Na}$ pesquisa realizada com os alunos do curso de medicina da cidade de Presidente Prudente ficou evidente a falta de conhecimento sobre a tecnologia Realidade Virtual e Realidade Aumentada pela maioria dos alunos o que não é uma surpresa levando-se em conta o alto custo da maioria dos dispositivos utilizados.

Dando continuidade a pesquisa seria importante a elaboração de um questionário sobre a utilização de simuladores ou técnicas de simulação médica e enviar aos responsáveis de todas as escolas médicas existentes no país com o objetivo de contribuir para um melhor conhecimento do ensino ministrado e motivar os estudantes para fazer uso dessas ferramentas. A divulgação da tecnologia pode contribuir e despertar o interesse para novas pesquisas na área médica e 
também, de alguma forma pressionar os responsáveis para a aquisição de novas aplicações para a utilização de simuladores médicos.

\section{REFERÊNCIAS}

Cardoso, Alexandre; Lamounier Jr., Edgard. Aplicações de RV e RA na Educação e Treinamento. In: Simpósio de Realidade Virtual e Aumentada, XI, 2009, Porto Alegre. Livro do Pré-Simpósio, Porto Alegre: SBC, 2009. Disponível em: http://www.ckirner.com/download/livros/SVR2009_ PreSimpósio.pdf. Acesso em: 30 out. 2011.

KIRNER, Claudio; SISCOUTO, Robson Augusto. Fundamentos de Realidade Virtual e Aumentada. In: SYMPOSIUM ON VIRTUAL AND AUGMENTED REALITY, IX, 2007, Petrópolis. Livro do Pré-Simpósio, Porto Alegre: SBC, 2007. Disponível em: http://www.de.ufpb.br/ labteve/publi/2007_svrps.pdf. Acesso em: 1 nov. 2011.

Montero, Edna Frasson de Souza; zanchet, Dinamar José. Realidade virtual e a medicina. Disponível em: <http://www.scielo.br/scielo.php?script= sci_arttext\&pid=S01026502003000500017>. Acesso em: 21 out. 2011.

Nunes, Fátima L. S.; Costa, Rosa M. E. M.; Oliveira, Ana Cláudia M. T. G.; Delfino, Sérgio R.; Pavarini, Larissa; Rodello, Ildeberto A.; Brega, José Remo F.; Sementille, Antônio C.. Aplicações Médicas usando Realidade Virtual e Realidade Aumentada. In: Symposium on Virtual and Augmented Reality, IX, 2007, Petrópolis. Livro do Pré-Simpósio, Porto Alegre: SBC, 2007. Disponível em: http://www.de.ufpb.br/ labteve/publi/2007_svrps.pdf. Acesso em: 1 nov. 2011.

Nunes, Fátima L. S.; Machado, Liliane S.; Costa, Rosa M. E. M.. RV e RA Aplicadas à Saúde. In: Simpósio de Realidade Virtual e Aumentada, XI, 2009, Porto Alegre. Livro do Pré-Simpósio, Porto Alegre: $\quad$ SBC, $2009 . \quad$ Disponível em: http://www.ckirner.com/download/livros/SVR2009_PreSimposio.pdf. Acesso em: 30 out. 2011.

Pontual, Jorge; Menezes, César. Tecnologia: Jornal da Globo faz você provar a realidade aumentada. Disponível em: < http://blogs.abril.com.br/ antenaparabolica/2009/06/tecnologiajornal-globo-faz-voce-provar-realidade-aumentada.html >. Acesso em 13 nov. 2009.

Silvano, Douglas Machado; Justino, Helder Espíndula; Freitas, Kelvin Teixeira; AGUIAR, Reinaldo de. Disponível em: < http://reiag.vilabol.uol.com.br/ completo.htm >. Acesso em: 14 nov. 2009.

Skinovsky, James; Chibata, Maurício; Siqueira, Daniel Emílio Dalledone. Realidade virtual e robótica em cirurgia - Aonde chegamos e para onde vamos? In Revista do Colégio Brasileiro de Cirurgiões: vol. 35, n. 5, sep./oct. 2008. Disponível em: <http://www.scielo.br/scielo.php?script=sci_arttext\&pid=S0100-69912008000500011>. Acesso em: 12 nov. 2011. 